\title{
Uso de tecnologías en el lugar de atención para el manejo de la pandemia por COVID-19 en Colombia
}

\author{
Diego Montenegro-López ${ }^{1}$
}

Forma de citar

Montenegro-López D. Uso de tecnologías en el lugar de atención para el manejo de la pandemia por COVID-19 en Colombia. Rev Panam Salud Publica. 2020;44:e97. https://doi.org/10.26633/RPSP.2020.97

RESUMEN

Objetivo. Proponer un modelo de atención en salud que integra tecnologías que pueden emplearse en el lugar de atención (point-of-care) y técnicas de inteligencia artificial.

Métodos. Se usó un modelo teórico en el que un millón de personas accedieron a la aplicación móvil CoronApp-Colombia, que recoge datos personales, signos, síntomas y nexos epidemiológicos compatibles con COVID-19. Empleando la información de la app se aplicaron técnicas de inteligencia artificial (ciencias de datos) en una sala situacional virtual.

Resultados. Los usuarios compatibles con COVID-19 serían priorizados y sometidos a una prueba de diagnóstico rápido para la búsqueda de anticuerpos anti-SARS-CoV-2. El tamizaje con la prueba de diagnóstico rápido permitiría detectar a las personas serorreactivas, en quienes se llevaría a cabo la confirmación diagnóstica mediante biología molecular (PCR). La información de los casos positivos confirmados por PCR se sometería nuevamente a técnicas de inteligencia artificial y estadística espacial para determinar los focos geográficos de infección. En estos focos se puede hacer búsqueda activa de contactos con los casos índices positivos y activar nuevamente la ruta de diagnóstico con la prueba de diagnóstico rápido y PCR.

Conclusión. Este escenario puede ser un camino útil para que los países de la región con plataformas tecnológicas para el diagnóstico por PCR débiles o ausentes puedan maximizar los recursos existentes, estimar el peso epidemiológico de la COVID-19 (infección, morbilidad, mortalidad y letalidad) en sus territorios y definir planes de contención, mitigación y control acordes a sus necesidades.

Palabras claves Pandemias; infecciones por coronavirus; coronavirus; salud pública; inteligencia artificial; monitoreo epidemiológico; pruebas serológicas; Colombia.

La pandemia por COVID-19 causada por el virus emergente SARS-CoV-2 ha infectado alrededor de 1 millón 700 mil personas en más de 180 países de todos los continentes y ha causado más de 102000 muertes $(1,2)$.

Desde la confirmación del primer caso a finales de diciembre del 2019 en China (3), hasta los tres primeros meses de la pandemia, los frentes políticos, científicos y tecnológicos se han focalizado en la preparación, contención y mitigación de la enfermedad, promoviendo principalmente el autocuidado (higiene) y el distanciamiento social (resguardo, cuarentena, distanciamiento físico y restricción de actividades cotidianas) (4). Paralelamente, se están buscando respuestas sobre el origen del virus, su patogenia, las vías de transmisión, el espectro clínico de la enfermedad, el desarrollo de técnicas y métodos de diagnóstico, y el desarrollo de tratamientos y vacunas que permitan disminuir el impacto de la pandemia $(5,6)$.

La búsqueda de técnicas y métodos de diagnóstico para la detección de infecciones es en la actualidad uno de los grandes desafíos, dado que se necesita con urgencia conocer el peso epidemiológico de la enfermedad, inclusive el número de personas infectadas, la morbilidad, la mortalidad y la tasa de letalidad. El conocimiento real de la epidemiología de la COVID-19 permitirá definir planes y políticas en salud pública acordes a la dimensión del problema. Para esto, la Organización Mundial de

\footnotetext{
1 Fundación Chilloa, Santa Marta, Colombia. $\triangle$ Diego Montenegro-López dc.montenegro85@gmail.com
} 
la Salud (OMS) ha hecho un llamado a que los países, además de promover el autocuidado y el distanciamiento social, refuercen sus capacidades en el diagnóstico masivo y oportuno, el aislamiento y la búsqueda de contactos. Estos aspectos son considerados fundamentales para responder a la pandemia $(4,7)$.

Para el diagnóstico se ha establecido como prueba de referencia la reacción en cadena de la polimerasa (PCR), que permite la detección directa del virus en pacientes en quienes se sospecha COVID-19 $(7,8)$. Dada la demanda excesiva de insumos y reactivos de PCR para COVID-19 en todo el mundo, estos se han agotado $(4,9)$. Esta situación es más evidente en los países de Latinoamérica, donde la falta de plataformas tecnológicas y reactivos para el diagnóstico está dificultando cumplir con el llamado de la OMS mencionado antes (9).

Ante esta necesidad, las pruebas inmunológicas para buscar anticuerpos IgM/IgA e IgG dirigidos contra el SARS-CoV-2 son una alternativa complementaria a la PCR y sirven para el rastreo de casos asintomáticos y contactos de los casos índices (confirmados por PCR), lo que permitiría encontrar, aislar e interrumpir la cadena de transmisión en menor tiempo.

La OMS y otros centros de investigaciones en salud han disponibilizado información de pruebas de diagnóstico rápido (PDR) para la detección de anticuerpos contra el coronavirus (10-12). Estas pruebas han sido validadas y priorizadas por los órganos oficiales dentro de los respectivos países productores.

Para integrar estas dos técnicas de diagnóstico y responder a las limitaciones presentes en los países latinoamericanos, se propone aquí un modelo teórico que permitiría responder al llamado de la OMS ("pruebas, pruebas y más pruebas") y hacer frente a la pandemia. Para este modelo teórico se ha seleccionado a Colombia por la dinámica en las políticas en salud pública con la que viene manejando la COVID-19.

Colombia ha reportado 608 casos confirmados y 6 muertes asociados a COVID-19 (13). El país cuenta con un único laboratorio para efectuar las PCR, ubicado en el Instituto Nacional de Salud (INS), Bogotá. Con la demanda creciente de pruebas, al 22 de marzo el INS ha capacitado a 22 laboratorios de salud pública y de centros de investigación del país para el diagnóstico de COVID-19 a través de PCR (9).

Actualmente es preocupante que los insumos necesarios para desarrollar las pruebas por PCR se encuentran agotados en los países de la región (9). El 25 de marzo el Ministerio de Salud de Colombia, apoyado por la Asociación Colombiana de Infectólogos y el INS, emitió la resolución 019, que permite aplicar pruebas de diagnóstico rápido para buscar anticuerpos como proceso de tamizaje previo a la PCR, que sigue siendo la prueba de referencia $(8,14)$.

En este contexto, es necesario definir los posibles modelos de atención en salud que permitan unir las pruebas de diagnóstico rápido y la PCR; el objetivo de este estudio es proponer un modelo que integra el concepto de atención en el lugar (point-ofcare) y técnicas de inteligencia artificial.

\section{MÉTODOS}

Desde una visión reduccionista, el abordaje de atención en el lugar busca que la atención médica y principalmente, el diagnóstico, sea de acceso inmediato, oportuno y con resultados en minutos, inclusive la atención directa en un laboratorio, un consultorio, el barrio, la vivienda o la cama del paciente. Su finalidad es garantizar el tratamiento oportuno y adecuado o las medidas correctivas acertadas en pro del bienestar del individuo y el beneficio colectivo (15-17). A pesar que este abordaje viene desde la década de los 80 (18), con los avances en la biotecnología, los sistemas de información geográfica y la inteligencia artificial, en la actualidad surge una visión holística e integradora entre salud y tecnología con implicaciones de mayor costo-efectividad (19-21). Este enfoque ha sido aplicado y recomendado por varios países de Asia para afrontar la COVID-19 (12,22).

\section{Propuesta de modelo de atención en el lugar}

El modelo teórico parte de las siguientes premisas: 1. De cada 100 personas sanas (sin contacto con el SARS-CoV-2) fuera del epicentro de la pandemia en Wuhan, China (3), que tengan contacto con casos humanos de COVID-19 (infectivos), por lo menos 50 podrán adquirir la infección (23); 2. De los 50 infectados con el coronavirus, 7 serán casos asintomáticos (no presentarán síntomas compatibles con COVID-19), entre 24 y 31 serán presintomáticos y entre 12 y 19 desarrollarán un cuadro agudo durante las dos primeras semanas de infección (sintomáticos) (23-27); y 3. Más del 90,0\% de las personas que tuvieron contacto con el SARS-CoV-2 desarrollarán anticuerpos contra el virus a partir del séptimo día de la infección primaria $(28,29)$.

El modelo de atención en el lugar consiste en seis etapas o filtros, cuatro de ellas de diagnósticos en series: dos de estas corresponden a diagnósticos por ciencia de datos y dos a diagnósticos biológicos (pruebas de diagnóstico rápido y PCR).

Etapa 1: Acceso a la aplicación móvil. Se basa en la hipótesis de que un millón de personas residentes en Colombia han tenido acceso a la aplicación móvil CoronApp-Colombia (https:/ / play.google.com/store/apps/details?id=co.gov.ins. guardianes\&hl=es-419). CoronApp-Colombia permite que los usuarios reporten su estado de salud a fin de identificar los lugares potenciales de circulación viral. Tras registrar los datos personales el usuario completa un cuestionario epidemiológico sobre los síntomas que presenta en el día de acceso y sus contactos en los últimos 14 días. La información obtenida puede ser analizada automáticamente por técnicas de ciencia de datos (Figura 1A).

Etapa 2: Ciencia de datos y estadística espacial. La información aportada por los usuarios de la app puede ser analizada por técnicas de aprendizaje automático (machine learning), análisis de árboles de decisión o redes neurales artificiales, entre otras técnicas de inteligencia artificial (30). Las técnicas de inteligencia artificial, combinadas con técnicas cartográficas, son herramientas útiles para buscar, predecir, vigilar y controlar focos de infecciones humanas (22,31-33). Se propone emplear estas técnicas porque permiten manejar mejor la cantidad de variables y el análisis de datos masivos en comparación con un agente humano.

En esta etapa se sugiere la creación de un centro o comité de inteligencia artificial virtual para COVID-19, que puede estar integrado por profesionales expertos del área de biomedicina (médicos, bacteriólogos, biólogos, etc.), de ingeniería computacional (estadísticos, sistemas, electrónicos, geógrafos, etc.) y del área humana (psicología, comunicación social, periodismo, etc.), que pueden converger en una sala situacional virtual. En esta sala virtual se puede analizar masivamente los datos y 
FIGURA 1. Modelo de atención en salud para la pandemia por COVID-19 en países con baja capacidad tecnológica y científica de diagnóstico

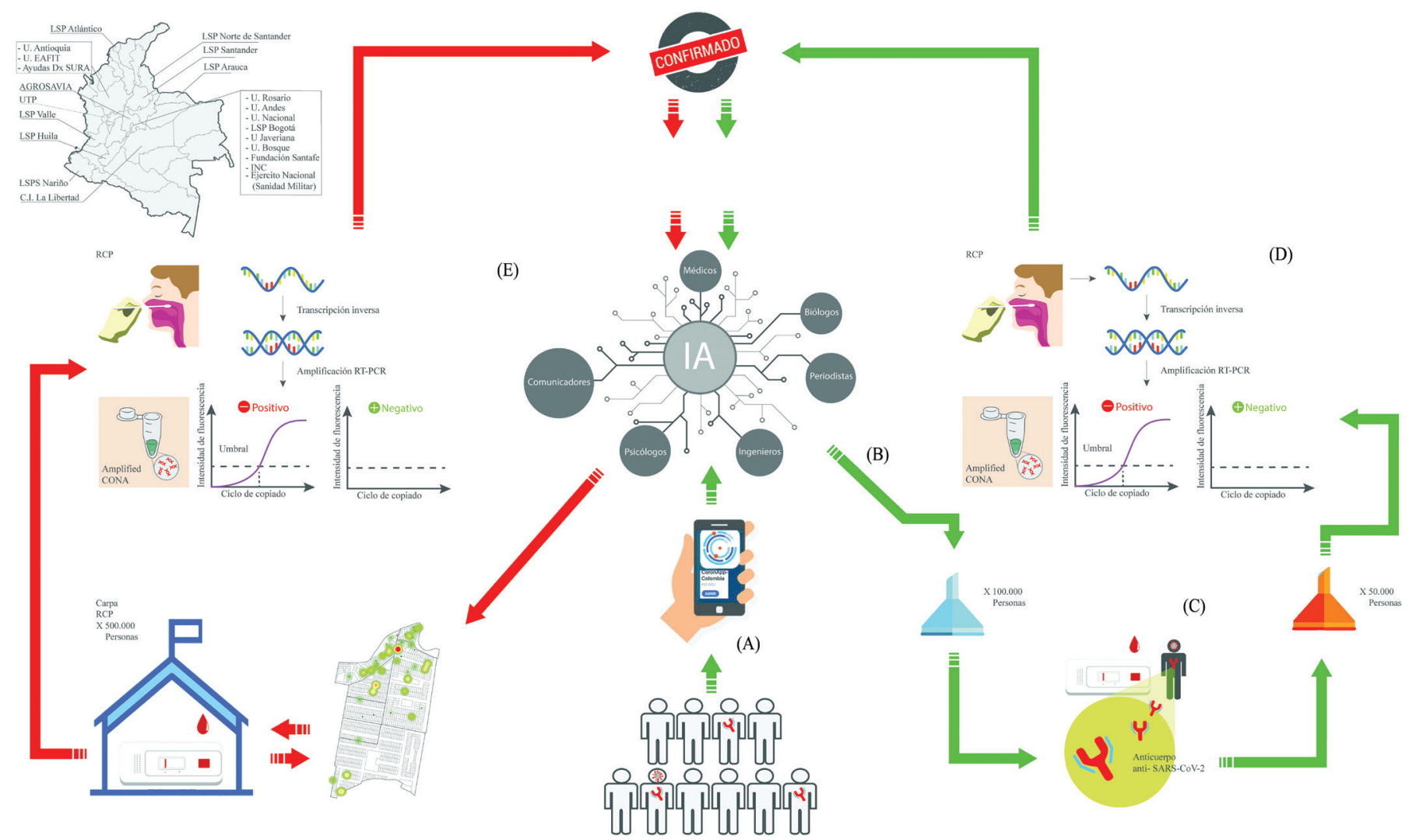

A. La población accede voluntariamente a la app (un millón de personas). Tanto los casos sintomáticos como los asintomáticos son registrados en bases de datos, inclusive su localización geográfica. B. Con ayuda de algoritmos matemáticos y minería de datos, las personas compatibles con COVID-19 son seleccionadas. Profesionales de la salud pueden añadir o excluir datos atípicos en una sala situacional virtual. Los profesionales biomédicos junto a los ingenieros direccionarán a los pacientes para que sean sometidos a una prueba de diagnóstico rápido y/o PCR, en un horario y fecha asignados. C. Lugares de realización de la prueba de diagnóstico rápida; en este momento puede notificarse al SIVIGILA. Los resultados y recomendaciones puede enviarse al paciente a través de la app. D. El tamizaje con la prueba de diagnóstico rápida permite detectar a las personas que han tenido contacto con elon proporcionada por los casos índices se establece un cerco epidemiológico y se activa una red de búsqueda activa de contactos con las personas infectados a través de pruebas de diagnóstico rápido.

hacer el primer filtro para la asignación de pruebas de diagnóstico rápido o asignación directa de $\mathrm{PCR}$, así como el manejo psicosocial de los casos compatibles con COVID-19 (Figura 1B).

Etapa 3: Prueba de diagnóstico rápido en el lugar de atención. Según la demanda observada en la etapa 2, es posible establecer lugares específicos para efectuar pruebas de diagnóstico rápido. En cada uno de ellos, profesionales del área de biomedicina administrarían las pruebas empleando un código único y con un horario estipulado de toma de muestra, asignados al usuario a través de la app. En este momento se puede iniciar la notificación al Sistemas de Vigilancia de Salud Pública de Colombia (SIVIGILA) (34), siguiendo los lineamientos de la entidad. En esta etapa los funcionarios de salud también pueden implementar el flujograma de diagnóstico establecido por la ACIN (8). Todos los pacientes que resulten serorreactivos para el coronavirus deben ser sometidos a la prueba confirmatoria con PCR (Figura 1C).

Etapa 4: Diagnóstico por PCR. Las personas serorreactivas deberán ser remitidas a los sitios adecuados para la toma de la muestra según las directrices del INS $(35,36)$. Las muestras se remitirán al laboratorio más próximo de la red nacional de Colombia habilitado para el diagnóstico de COVID-19 (9) (Figura 1D).

Etapa 5: Confirmación de casos. Tras aproximadamente 48 horas desde la toma de muestra, el SIVIGILA contará con la confirmación de los casos. Estos casos confirmados activarán una ruta inversa a la empleada en la etapa 2 , con aplicación de técnicas cartográficas y de estadística espacial; esto permitirá definir los clusters epidémicos para encontrar otras personas infectadas, como se ha implementado en Asia (37-39) (Figura 1E).

Etapa 6: Cerco epidemiológico. Esta etapa se concentrará en la búsqueda activa de los contactos de los casos confirmados; las pruebas de diagnóstico rápido pueden focalizarse a nivel de la vivienda o la manzana, según los mapas probabilísticos generados antes.

\section{RESULTADOS Y DISCUSIÓN}

Con la implementación del modelo propuesto o uno similar, Colombia y los países de la región podrían conocer el impacto epidémico real de la enfermedad y efectuar diagnósticos más 
rápidos y costo-efectivo para afrontar la pandemia. La costo-efectividad de las pruebas de diagnóstico rápido frente a la PCR puede asociarse a que esta última requiere infraestructuras físicas complejas, equipos e insumos de laboratorio de mayor costo y personal altamente especializado. Además, la eficacia de la PCR depende en gran medida de la carga viral y de la presencia de cantidades suficientes de virus en el sitio y momento de la toma de muestra, así como del procesamiento y transporte de la muestra; estos factores pueden ocasionar falsos negativos, con pérdida de la oportunidad de aislar al paciente e interrumpir la cadena de transmisión viral (29).

Según el reporte de la Procuraduría de Colombia (40), el costo de cada PCR es de 380 mil pesos colombianos (aprox. USD \$93,99; TRM USD = 4042 pesos colombianos). Hasta el 27 de marzo el INS ha realizado 11384 pruebas de PCR con 5,9\% de positividad para el SARS-CoV-2 (13); estos datos permiten dimensionar el costo actual para el sistema de salud relacionado con la captación de los casos positivos de COVID-19.

Algunos estudios, empleando técnicas ELISA, están demostrando que la respuesta humoral al coronavirus (específicamente, la producción de anticuerpos $\operatorname{IgM} / \operatorname{IgA}$ e IgG después de 5 y 14 días del inicio de los síntomas, respectivamente) puede detectarse en $78,0 \%$ de los casos confirmados por PCR y 93,0\% de los casos sospechosos (29). Este mismo estudio determinó que la tasa de detección positiva aumenta a 98,6\% cuando se combina la detección de IgM con PCR para cada paciente en comparación con una sola prueba de PCR $(51,9 \%)$. Por lo tanto, se demuestra que está ocurriendo seroconversión en los pacientes infectados con el SARS-CoV-2 y que es viable la implementación de estudios serológicos combinados con estudios moleculares. Recientemente se demostró una sensibilidad del $88,7 \%$ y una especificidad del $90,6 \%$ de una prueba de diagnóstico rápido (resultados en 15 minutos), usando como método de referencia la PCR (28).

Si se considera el tiempo que conlleva obtener los resultados de las PCR en Colombia (mayor a 14 días) (40) y la alta proporción de casos negativos (94,0\%) (13), la integración de estas dos técnicas con inteligencia artificial, además de disminuir drásticamente el tiempo en entrega de resultados, puede ser más costo-efectiva al filtrar o disminuir el número de pacientes sospechosos que no están infectados con el SARS-CoV-2 e interrumpir la cadena de transmisión en menor tiempo. Una ventaja adicional es que el diagnóstico se realiza con una pequeña gota de sangre y el personal responsable de la aplicación de la prueba de diagnóstico rápido tiene menor exposición a adquirir el virus en comparación con la toma de muestras para PCR.

La principal limitación del modelo de atención de salud propuesto es la validación de la reproducibilidad de las pruebas de diagnóstico rápido. E1 28 de marzo las entidades competentes de Colombia aprobaron una prueba diagnóstica rápida (https: / / caracol.com.co/radio/2020/03/29/salud/1585439681_292078. $\mathrm{html}$ ), pero no se encontró especificaciones técnicas de la misma, lo que imposibilitó evaluar su reproducibilidad. Si Colombia y los países de la región deciden adaptar o adoptar un modelo como el propuesto, deben validar las diferentes pruebas de diagnóstico rápido disponibles en el mercado y priorizarlas según los resultados encontrados. Una limitación adicional se asocia con al número de casos asintomáticos y presintomáticos que pueden no ser detectados durante el primer filtro (Figura 1B), pero que puede ser abordado si se lleva a cabo un tamizaje al azar a por lo menos 10,0\% de las personas sin síntomas. Las técnicas de inteligencia artificial junto a los agentes humanos de la sala virtual, pueden priorizar esas personas. Esta limitación también puede reducirse si se agiliza la entrega de resultados de las PCR y la ruta inversa (Figura 1E).

Finalmente se sugiere que, si se implementan pruebas de diagnóstico rápido para COVID-19 en los países de la región, por lo menos el $10,0 \%$ de estas se reserve para el recurso humano del área de biomedicina que actualmente se encuentra en el frente de atención y diagnóstico. Esto puede brindar información del estado serológico de los profesionales y proporcionar más confianza y seguridad en su trabajo frente a la pandemia del COVID-19.

Agradecimientos. A Daniel Noriega por el apoyo en la realización gráfica del modelo de atención.

Financiación. El autor.

Conflicto de interés. Ninguno declarado.

Declaración. Las opiniones expresadas en este manuscrito son responsabilidad del autor y no reflejan necesariamente los criterios ni la política de la RPSP/PAJPH y / o de la OPS.

\section{REFERENCIAS}

1. World Health Organization. Novel coronavirus (COVID-19) situation [Internet]. World Health Organization. 2020 [citado 2020 Mar 24]. Disponible en: https://experience.arcgis.com/experience/ 685d0ace521648f8a5beeeee1b9125cd

2. Johns Hopkins University. Coronavirus Resource Center [Internet]. Johns Hopkins University and Medicine. 2020 [citado 2020 Mar 28]. Disponible en: https: / / coronavirus.jhu.edu/map.html

3. Huang C, Wang Y, Li X, Ren L, Zhao J, Hu Y, et al. Clinical features of patients infected with 2019 novel coronavirus in Wuhan, China. Lancet. 2020;395(10223):497-506.

4. Organización Mundial de la Salud. Alocución de apertura del Director General de la OMS en la rueda de prensa sobre la COVID-19 celebrada el 16 de marzo de 2020 [Internet]. Organización Mundial de la Salud; 2020 [citado 2020 Mar 20]. Disponible en: https:/ / www. who.int/es/dg/speeches/detail/who-director-general-s-openingremarks-at-the-media-briefing-on-covid-19---16-march-2020
5. Cascella M, Rajnik M, Cuomo A, Dulebohn SC, Di Napoli R. Features, Evaluation and Treatment Coronavirus (COVID-19) [Internet]. StatPearls. 2020 [citado 2020 Mar 25]. Disponible en: http://www. ncbi.nlm.nih.gov/pubmed/32150360

6. Yuen K-S, Ye Z-W, Fung S-Y, Chan C-P, Jin D-Y. SARS-CoV-2 and COVID-19: The most important research questions. Cell Biosci [Internet]. 2020 [citado 2020 Mar 25];10(1):40. Disponible en: https://cellandbioscience.biomedcentral.com/articles/10.1186/ s13578-020-00404-4

7. World Health Organization. Laboratory testing for 2019 novel coronavirus (2019-nCoV) in suspected human cases [Internet] 2020 [citado 2020 Mar 27]. Disponible en: https://www.who.int/ publications-detail/laboratory-testing-for-2019-novel-coronavirusin-suspected-human-cases-20200117

8. Asociación Combiana de Infectología. Consenso colombiano de atención, diagnóstico y manejo de la infección por SARS-CoV-2/ 
COVID-19 en estableciemientos de atención de la Salud [Internet]. Infectio. 2020;24(3). Disponible en: http://www.revistainfectio. org/index.php/infectio/article/view/851/899

9. Instituto Nacional de Salud. INS Colombia entrena a universidades para el diagnóstico del COVID-19 [Internet]. INS; 2020 [citado 2020 Mar 26]. Disponible en: https://www.ins.gov.co/ Noticias/Paginas/INS-Colombia-habilitará-a-universidades-para-eldiagnóstico-del-COVID-19.aspx

10. Foundation for Innovative New Diagnostics. SARS-CoV-2 diagnostic pipeline [Internet]. FIND; 2020 [citado 2020 Mar 29]. Disponible en: https:/ / www.finddx.org/covid-19/pipeline/

11. World Health Organization. Update on COVID-19 in vitro diagnostics listed by National Regulatory Authorities in IMDRF jurisdictions [Internet]. [citado 2020 Mar 28]. Disponible en: https: / / www.who.int/diagnostics_laboratory/200408_imdrf_covid19_ listing_update_8_april_2020.pdf?ua $=1$

12. Sheridan C. Fast, portable tests come online to curb coronavirus pandemic. Nat Biotechnol [Internet]. 2020 Mar 23 [citado 2020 Mar 27]; Disponible en: http:/ / www.ncbi.nlm.nih.gov/pubmed/32203294

13. Instituto Nacional de Salud. Coronavirus (COVID 2019) en Colombia [Internet]. INS; 2020 [citado 2020 Mar 27]. Disponible en: https://www.ins.gov.co/Noticias/Paginas/Coronavirus.aspx

14. Ministerio de Salud. Detección Temprana SARS CoV-2/ COVID-19 [Internet]. MInisterio de Salud y de la Proteccion Social Colombia: 25 de marzo 2020; 2020. Disponible en: https:/ / www.minsalud.gov. co/Documents/20200325_Circular_019.pdf

15. Kost GJ. Guidelines for point-of-care testing: Improving patient outcomes. Am J Clin Patholo. 1995;104.

16. Summers $\mathrm{SH}$, Harmening D, Lunz ME. Who performs point-of-care testing? Lab Med. 1998;29(2):85-8.

17. Kozel TR, Burnham-Marusich AR. Point-of-care testing for infectious diseases: Past, present, and future. J Clin Microbiol. 2017;55:2313-20.

18. Morrison EM, Luft HS. Health maintenance organization environments in the 1980s and beyond. Health Care Financ Rev. 1990;12(1):81-90.

19. St John A, Price CP. Existing and Emerging Technologies for Pointof-Care Testing. Clin Biochem Rev. 2014;35(3):155-67. Disponible en: http://www.ncbi.nlm.nih.gov/pubmed/25336761 [citado 2020 Mar 27]

20. Jang SM, Lee K, Hong Y-J, Kim J, Kim S. Economic Evaluation of Robot-Based Telemedicine Consultation Services. Telemed e-Health. 2020 Jan 22 [citado 2020 Mar 27]; Disponible en: http:/ / www.ncbi. nlm.nih.gov/pubmed/31971885

21. Christodouleas DC, Kaur B, Chorti P. From Point-of-Care Testing to eHealth Diagnostic Devices (eDiagnostics). ACS Cent Sci. 2018;4(12):1600-16.

22. Wang CJ, Ng CY, Brook RH. Response to COVID-19 in Taiwan: Big Data Analytics, New Technology, and Proactive Testing. JAMA. 2020;323(14):1341-2.

23. Du Z, Wang L, Cauchemez S, Xu X, Wang X, Cowling BJ, et al. Risk for Transportation of 2019 Novel Coronavirus Disease from Wuhan to Other Cities in China. Emerg Infect Dis. 2020;26(5).

24. Nishiura H, Kobayashi T, Suzuki A, Jung S-M, Hayashi K, Kinoshita R, et al. Journal Pre-proof Estimation of the asymptomatic ratio of novel coronavirus infections (COVID-19). Int J Infect Dis. 2020 [citado 2020 Abr 6]. Disponible en: https://doi.org/10.1016/j. ijid.2020.03.020

25. Mizumoto K, Kagaya K, Zarebski A, Chowell G. Estimating the asymptomatic proportion of coronavirus disease 2019 (COVID-19) cases on board the Diamond Princess cruise ship, Yokohama, Japan, 2020. Eurosurveillance. 2020;25(10).

26. Ganyani T, Kremer C, Chen D, Torneri A, Faes C, Wallinga J, et al. Estimating the generation interval for COVID-19 based on symptom onset data. medRxiv. 2020 Mar 8;2020.03.05.20031815.

27. Du Z, Xu X, Wu Y, Wang L, Cowling BJ, Meyers LA. Serial Interval of COVID-19 among Publicly Reported Confirmed Cases. Emerg Infect Dis. 2020;26(6). [citado 2020 Abr 6]; Disponible en: http:// www.ncbi.nlm.nih.gov/pubmed/32191173

28. Li Z, Yi Y, Luo X, Xiong N, Liu Y, Li S, et al. Development and Clinical Application of A Rapid IgM-IgG Combined Antibody Test for
SARS-CoV-2 Infection Diagnosis. J Med Virol. 2020 Feb 27;10.1002/ jmv.25727. [citado 2020 Abr 16]; Disponible en: https://online library.wiley.com/doi/abs/10.1002/jmv.25727

29. Guo L, Ren L, Yang S, Xiao M, Chang D, Yang F, et al. Profiling Early Humoral Response to Diagnose Novel Coronavirus Disease (COVID-19). Clin Infect Dis. 2020;ciaa310 [citado 2020 Abr 16] Disponible en: https://academic.oup.com/cid/article/doi/10.1093/ cid/ciaa310/5810754

30. Stuart R, Norvig P. Artificial Intelligence. In: Stuart R, Norvig P, editors. Artificial Intelligence: A Modern Approach [Internet]. 2nd ed. New Jersey: Pearson Education; 2003. Disponible en: http:// coltech.vnu.edu.vn/ sonpb/AI/AIMA.pdf

31. Montenegro D, de Mello FL, Giordano Dias CM, Almeida P, Araújo M, Magalhães MA, et al. Evaluating the Surveillance System for Spotted Fever in Brazil Using Machine-Learning Techniques. Front Public Health. 2017;5:323. [citado 2017 Dic 13] Disponible en: http:/ / journal.frontiersin.org/article/10.3389/fpubh.2017.00323/full

32. Montenegro D, Cunha AP da, Ladeia-Andrade S, Vera M, Pedroso M, Junqueira A, et al. Multi-criteria decision analysis and spatial statistic: an approach to determining human vulnerability to vector transmission of Trypanosoma cruzi. Mem Inst Oswaldo Cruz. 2017; 112(10):709-18. [citado 2017 Oct 3] Disponible en: http://www.scielo.br/scielo.php?script=sci_arttext\&pid= S0074-02762017001000709\&lng=en\&tlng=en

33. Montenegro D, Cunha A Da, Machado I, Duraes L, De Oliveira S, Pedroso M, et al. One world, one health challenge: the holistic understanding of Rickettsiosis integrating multicriteria analysis techniques and spatial statistics. En: Jorge R, editor. Spatial Analysis, Modelling and Planning. 1st ed. London: IntechOpen; 2018. p. 20.

34. Instituto Nacional de Salud. Vigilancia Rutinaria, 2016 [citado 2016 May 6]. Disponible en: http://www.ins.gov.co/lineasde-accion/Subdireccion-Vigilancia/sivigila/Paginas/vigilanciarutinaria.aspx

35. Instituto Nacional de Salud. Lineamiento de toma de muestras por vía respiratoria. Bogotá: Instituto Nacional de Salud; 2006 [citado 2020 Mar 28]. Disponible en: https://www.ins.gov.co/Noticias/ Coronavirus/Toma de muestras para un correcto diagnóstico de los virus respiratorios- INS.mp4

36. Instituto Nacional de Salud. Lineamientos para la vigilancia por Laboratorio de virus respiratorios. Bogotá; INS; 2006 [citado 2020 Mar 28]. Disponible en: https://www.ins.gov.co/Noticias/ Coronavirus/Lineamientos para la vigilancia por Laboratorio de virus respiratorios 06.03.20.pdf

37. Pung R, Chiew CJ, Young BE, Chin S, I-C Chen M, Clapham HE, et al. Investigation of three clusters of COVID-19 in Singapore: implications for surveillance and response measures. Lancet. 2020;395:1039 [citado 2020 Mar 24]; Disponible en: http://www. ncbi.nlm.nih.gov/pubmed/32192580

38. Liu YF, Li JM, Zhou PH, Liu J, Dong XC, Lyu J, et al. [Analysis on cluster cases of COVID-19 in Tianjin]. Zhonghua Liu Xing Bing Xue Za Zhi. 2020; 41(5):654-7. [citado 2020 Mar 28]; Disponible en: http:/ / www.ncbi.nlm.nih.gov/pubmed/32213269

39. Gan H, Zhang Y, Yuan M, Wu XY, Liu ZR, Liu M, et al. [Epidemiological analysis on 1052 cases of COVID-19 in epidemic clusters] Zhonghua Liu Xing Bing Xue Za Zhi. 2020;41(5):E027. Mar 26 [citado 2020 Mar 28]; Disponible en: http://www.ncbi.nlm.nih. gov/pubmed /32213270

40. SEMANA. Procuraduría halló anomalías en el control de calidad de pruebas del covid-19. Revista Semana [Internet]. 2020 [citado 2020 Mar 29]; Disponible en: https://www.semana.com/nacion/ articulo/procuraduria-hallo-anomalias-en-el-control-de-calidadde-pruebas-del-covid-19/659142

Manuscrito recibido el 29 de marzo de 2020. Aceptado para su publicación, tras revisión, el 4 de junio de 2020. 


\section{Use of point-of-care technologies for the management of the COVID-19 pandemic in Colombia}

ABSTRACT Objective. To propose a health care model that integrates point-of-care technologies and artificial intelligence for the management of the COVID-19 pandemic.

Methods. A theoretical model was used in which one million people accessed the mobile application CoronApp-Colombia, which collects personal data, signs, symptoms and epidemiological links compatible with COVID-19. With the information from the app artificial intelligence techniques (data science) were applied in a virtual situation room.

Results. Users compatible with COVID-19 were prioritized and subjected to a rapid diagnostic test for antiSARS-CoV-2 antibodies. Screening with the rapid diagnostic test would allow detection of sero-reactive individuals, for whom diagnostic confirmation would be carried out using molecular biology (PCR). Information from positive cases confirmed by PCR would be re-screened using artificial intelligence and spatial statistical techniques to identify geographical foci of infection. These foci could be actively searched for contacts with positive index cases and the diagnostic route would be followed again using the rapid diagnostic test and PCR.

Conclusion. This model may be useful for countries in the region with weak or absent technological platforms for PCR diagnosis to maximize existing resources, estimate the epidemiological burden of COVID-19 (infection, morbidity, mortality and lethality) and implement containment, mitigation and control plans according to their needs.

Keywords Pandemics; coronavirus infections; coronavirus; public health; artificial intelligence; epidemiological monitoring; serologic tests; Colombia. 